\title{
Emotional dysfunction in schizophrenia spectrum psychosis: the role of illness perceptions
}

\author{
P. W. B. WATSON ${ }^{1 *}$, P. A. GARETY ${ }^{1}$, J. WEINMAN ${ }^{1}$, G. DUNN ${ }^{2}$, \\ P. E. BEBBINGTON ${ }^{3}$, D. FOWLER ${ }^{4}$, D. FREEMAN ${ }^{1}$ AND E. KUIPERS \\ ${ }^{1}$ King's College London, Department of Psychology, Institute of Psychiatry, London, UK; \\ ${ }^{2}$ School of Epidemiology and Health Sciences, University of Manchester, Stopford Building, \\ Manchester, UK; ${ }^{3}$ Department of Mental Health Sciences, University College London, London, UK; \\ ${ }^{4}$ School of Health Policy and Practice, University of East Anglia, Norwich, UK
}

\begin{abstract}
Background. Assessing illness perceptions has been useful in a range of medical disorders. This study of people with a recent relapse of their psychosis examines the relationship between illness perception, their emotional responses and their attitudes to medication.
\end{abstract}

Method. One hundred patients diagnosed with a non-affective psychotic disorder were assessed within 3 months of relapse. Measures included insight, self-reported illness perceptions, medication adherence, depression, self-esteem and anxiety.

Results. Illness perceptions about psychosis explained 46, 36 and $34 \%$ of the variance in depression, anxiety and self-esteem respectively. However, self-reported medication adherence was more strongly associated with a measure of insight.

Conclusions. Negative illness perceptions in psychosis are clearly related to depression, anxiety and self-esteem. These in turn have been linked to symptom maintenance and recurrence. Clinical interventions that foster appraisals of recovery rather than of chronicity and severity may therefore improve emotional well-being in people with psychosis. It might be better to address adherence to medication through direct attempts at helping them understand their need for treatment.

\section{INTRODUCTION}

Psychosis is seen increasingly as the unusual outcome of a concatenation of interacting cognitive and emotional processes that themselves lie within the normal range (Johns \& van Os, 2001; Freeman et al. 2005). Appraisal is central to cognitive models of the positive symptoms of psychosis (e.g. Garety et al. 2001; Morrison, 2001; Byrne et al. 2003). The concept of 'illness appraisal' or 'illness perception' can broadly be defined as a collection of beliefs relating to how individuals make sense of, and understand, their health status (see Lobban et al. 2003). One

\footnotetext{
* Address for correspondence: Philip W. B. Watson, Department of Psychology, PO 77, Henry Wellcome Building, Institute of Psychiatry, De Crespigny Park, London SE5 8AF, UK.

(Email: P.Watson@iop.kcl.ac.uk)
}

form of appraisal concerns the experience of the psychosis itself, whether seen as an illness or otherwise. This has been postulated to influence the maintenance and recurrence of psychotic symptoms. Appraisals are generally held to do this through their effect on coping responses (including medication adherence), emotional impact and cognitive processes.

Insight is the conventional way of conceptualizing how people with a psychiatric illness, particularly schizophrenia, evaluate their experience of it. It implies a degree of acceptance of the idea of illness. Many studies have highlighted the relationship between insight and clinical outcome, possibly mediated through engagement with treatment (Ghaemi \& Pope, 1994; Rathod et al. 2005). However, several authors (David, 1998; Cooke et al. 2005) have 
reported that standard measures of insight are only modestly associated with outcome. David (1998) also suggested that emotional dysfunction in schizophrenia may be related to appraisal of the personal and social consequences of severe mental illness rather than mere acknowledgment of the illness itself. This is essentially the concept of illness perception, a form of meta-cognition applicable to both physical and mental disorders.

In recent years, illness perception has been investigated in a wide range of medical conditions. Leventhal et al. (1984) proposed that people faced with a health threat assume the role of an 'active problem solver', in that they formulate a cognitive representation of their illness based on five core constructs. These are identity (the symptoms and label associated with the illness), cause (the aetiological attribution of the illness), cure/control (the extent to which they think their condition is amenable to cure or control), consequences (the personal, social and financial consequences of the illness) and timeline (how long they believe their illness will last). The model postulates that these cognitions will predict the practical and emotional responses to a health threat. Weinman et al. (1996) developed the Illness Perception Questionnaire (IPQ) to measure the illness perceptions in the model proposed by Leventhal et al. (1984). Bishop and Converse (1986) confirmed the validity and consistency of these illness constructs, and a recent meta-analysis of 45 studies provided confirmation of the key components and predictions within the model (Hagger \& Orbell, 2003).

Leventhal's model emphasizes that the importance of the emotional response to a health threat comes from its effects on practical coping responses. A recent meta-analysis by DiMatteo et al. (2000) provides support for this view; depressed mood made it three times less likely that medical patients would take medication.

In psychosis, however, there have been very few investigations of the role of patients' illness appraisals. One exception was the study by Iqbal et al. (2000), who used the Personal Beliefs and Illness Questionnaire (Birchwood et al. 1993) to assess 105 patients presenting with schizophrenia. They concluded that postpsychotic depression results from appraisal of illness, and that depression then leads to more pessimistic illness appraisal.

Although the application of the IPQ to psychosis is in its infancy, the predicted relationships between illness perceptions and emotional dysfunction have been reported (Barrowclough et al. 2001; Jolley \& Garety, 2004; Lobban et al. 2004). As illness perceptions seem to be amenable to manipulation (Petrie et al. 2002), this might pave the way for targeted cognitive interventions to promote more adaptive coping strategies and to enhance clinical outcome.

Individuals with psychosis usually experience some form of emotional dysfunction; for instance, clinical depression, low self-esteem and anxiety are common, and they are highly correlated (Freeman \& Garety, 2003). Birchwood (2003) postulated three possible origins of emotional dysfunction in individuals presenting with a first episode of psychosis: (i) those intrinsic to psychosis, (ii) the result of developmental anomaly and trauma or (iii) a psychological reaction to psychosis. It is the third pathway that is of particular relevance here. For example, recognition of the consequences associated with psychosis, such as the loss of social goals, roles and status, might well lead to post-psychotic depression and hopelessness (Iqbal et al. 2000). A perceived loss of control over the illness also appears to be predictive of demoralization and depression (Birchwood et al. 1993). The emphasis here is thus on the individual's appraisal of their illness.

A recent study by Lobban et al. (2004) examined relationships between illness perceptions and anxiety and depression alongside a number of other outcome variables in individuals with psychosis. We wanted to explore further whether these associations are stronger than relationships between emotional dysfunction and insight. This would test the "emotional response' pathway of the self-regulatory model of Leventhal et al. (1984) and also test the predictions of the cognitive model of positive symptoms of psychosis (Garety et al. 2001). Moreover, given that the first line of treatment in psychosis is medication, we aimed to test the hypothesized link between illness appraisals and coping in terms of adherence behaviour in the context of Leventhal's model.

Therefore, the present cross-sectional study was driven by the notion that incorporating 
illness perception constructs into a cognitive model of psychosis might enhance our understanding of emotional dysfunction in people experiencing a relapse of psychosis. We hypothesized that:

(1) illness perceptions would show a stronger association with emotional dysfunction than measures of insight. Specifically, apprehensions of a longer timeline, worse consequences, a heightened perception of symptoms and a low sense of control would be associated with higher levels of depression and anxiety, and lower levels of self-esteem;

(2) illness perceptions would be associated more strongly with a self-reported measure of medication adherence than insight.

We also investigated the association between depression and self-reported medication adherence. Given the limited amount of research investigating the link between causal attributions and behavioural and emotional responses to psychosis, we conducted exploratory analyses of putative causal factors and their relation to our dependent variables.

\section{METHOD}

\section{Sample}

The study sample consisted of participants recruited for the Psychological Prevention of Relapse in Psychosis (PRP) Trial (ISRCTN83557988). The PRP is a UK multi-centre randomized controlled trial of cognitive behaviour therapy and family intervention for nonaffective psychosis, designed to test hypotheses both about outcome and about the psychological processes associated with psychosis. Studies of psychological processes in psychosis were incorporated into the baseline assessment of participating patients before randomization into the trial took place.

The trial was located in four National Health Service (NHS) Trusts in London and East Anglia. In each Trust, recruitment was from specified clinical teams, both in-patient and outpatient services. These were approached at least fortnightly for patients with psychosis who were relapsing. Patients meeting the eligibility criteria were asked to provide informed consent for participation.
Participants were recruited at the time of a relapse in positive symptoms, either from a previously recovered state or from a state of persisting symptoms. For those with persistent symptoms, a significant exacerbation in positive symptoms was required. The inclusion criteria were: current diagnosis of non-affective psychosis (schizophrenia, schizo-affective psychosis, delusional disorder; ICD-10, F20); age 18-65 years; second or subsequent episode starting not more than 3 months before induction; and a rating of at least 4 (moderate severity) on at least one positive psychotic symptom of the Positive and Negative Syndrome Scale (Kay et al. 1991) at first assessment.

The first 100 participants in the PRP trial who had completed all the measures required for this study were included.

\section{Measures \\ Insight - Scale of Unawareness of Mental Disorder (SUMD; Amador et al. 1993)}

The development of this measure was based on the acknowledgement that insight is a complex phenomenon comprising a number of component dimensions and that degrees of insight are therefore possible. A series of questions were asked in order to rate the first five items of this measure. The items were (i) awareness of mental disorder; (ii) awareness of the achieved effects of medication; (iii) awareness of the social consequences of mental disorder; (iv) awareness/attribution of hallucinations; and (v) awareness/attribution of delusions. These items were chosen to measure the core aspects of insight described by Amador \& David (1998), and to assess insight in relation to the main positive symptoms of psychosis (i.e. hallucinations and delusions). Each item was rated on a five-point scale, whereby a score of 1 represented awareness and 5 represented unawareness. The ratings from these five items were summed, pro-rated if necessary, divided by 5 and reverse scored to produce a mean insight score, where a score of 1 represented poor insight, and 5, good insight.

\section{Illness Perception Questionnaire (IPQ; Weinman et al. 1996)}

The IPQ consists of items measuring five core illness constructs: identity, cause, consequences, 
cure/control and timeline. The IPQ measure is considered to be a reliable and valid tool with which to assess illness perceptions. The measure used in the present study was a version of the original IPQ modified by Weinman and Garety for use in psychosis samples and subsequently piloted (Jolley \& Garety, 2004). The consequences, timeline and cure/control constructs were measured by seven, three and six items, respectively, in accordance with the original IPQ of Weinman et al. (1996). Eleven attribution items were included in the questionnaire. Each IPQ item (excluding perceived symptom items) was measured on a scale of $1-5$, representing strongly disagree to strongly agree respectively.

The modified IPQ lists 18 symptoms in the identity (perceived symptom) component. However, in the current analysis we used only the six items specific to positive symptoms of psychosis: (i) seeing images; (ii) feeling confused; (iii) hearing voices; (iv) having paranoid thoughts; (v) holding beliefs not shared by others; and (vi) feeling that one's mind is being controlled. Negative symptoms were not included because of the correlation between negative symptoms and depression. For each item, the response format was $0-3$, with a score of 0 representing 'never' and 3 representing 'all the time'. Therefore, the mean composite identity score had a range of $0-3$.

\section{Rosenberg Self-Esteem Scale-(RSE;} Rosenberg, 1965)

The RSE consists of 10 items, each measured on a four-point scale from strongly agree to strongly disagree. After reverse scoring, the items were totalled and divided by 10 to produce a mean self-esteem score, where a high score represents low self-esteem.

\section{Beck Depression Inventory-II (BDI-II; Beck et al. 1996)}

This established measure consists of 21 items each measured on a scale of $0-3$. Therefore, the total BDI-II score ranges from 0 to 63, with a high score representing more symptoms. Depression is measured in relation to the previous 2 weeks. Birchwood et al. (2000) reported a high correlation $(r=0.91)$ between the BDI and the interview-based Calgary Depression Scale for Schizophrenia (Addington et al. 1993), confirming that the BDI can be used to assess depression in psychosis.

Beck Anxiety Inventory (BAI; Beck et al. 1988)

This measure consists of 21 items, each measuring common anxiety symptoms. The total anxiety score ranges from 0 to 63 , with a higher score indicating more anxiety. Anxiety is measured in relation to the previous week.

\section{Medication Adherence Rating Scale (MARS; Thompson et al. 2000)}

This self-report measure consists of 10 dichotomous yes/no items, measuring behavioural aspects of adherence and attitudes towards medication. Total scores ranged from 0 to 10 , where 0 and 10 represent low and high likelihood of adherence to medication respectively. As many participants were in hospital during the assessment phase, these individuals were asked to complete the behaviour component (four items) of this questionnaire based on the time immediately prior to their admission. During the development of this measure, the MARS was significantly correlated with two other self-report measures (Hogan et al. 1983; Morisky et al. 1986), thus demonstrating considerable concurrent validity. Internal reliability and test-retest reliability were also shown to be satisfactory. Moreover, significant correlation has been shown between this measure and blood levels of prescribed mood stabilizers $(r=0 \cdot 60$, $p<0 \cdot 05)$.

\section{Data analysis}

Analyses were conducted using SPSS for Windows version 12.0 (SPSS Inc., Chicago, IL, USA). Bivariate correlations measured the strength of associations between the independent variables (insight and IPQ constructs) and the dependent variables (depression, anxiety, self-esteem and medication adherence). The IPQ causal attribution items were analysed separately. Independent variables that correlated with the dependent variables at the $p<0.05$ level were entered into simultaneous multiple regression analyses. It is generally accepted that for each independent variable entered into a regression equation, between five and 20 participants are required. The number of participants in the present study exceeded the number required to obtain $80 \%$ power, assuming the 
Table 1. Mean, standard deviations and internal reliability statistics of independent and dependent variables

\begin{tabular}{|c|c|c|c|c|}
\hline & $\begin{array}{l}\text { Number } \\
\text { of items }\end{array}$ & $\begin{array}{c}\text { Internal } \\
\text { reliability } \\
\text { (Cronbach's } \alpha \text { ) }\end{array}$ & $\begin{array}{c}\text { Mean } \\
(n=100)\end{array}$ & $\begin{array}{l}\text { Standard } \\
\text { deviation }\end{array}$ \\
\hline \multicolumn{5}{|l|}{ Independent variables } \\
\hline Insight & 5 & $0 \cdot 82^{\mathrm{a}}$ & $3 \cdot 02$ & $1 \cdot 21$ \\
\hline Identity & 6 & N.A. & $1 \cdot 20$ & $0 \cdot 67$ \\
\hline Consequences & 7 & $0 \cdot 64$ & $3 \cdot 70$ & $0 \cdot 70$ \\
\hline Cure/Control & 6 & $0 \cdot 64$ & $3 \cdot 34$ & $0 \cdot 74$ \\
\hline Timeline & 3 & $0 \cdot 86$ & $3 \cdot 20$ & $1 \cdot 14$ \\
\hline \multicolumn{5}{|l|}{ Dependent variables } \\
\hline Anxiety (BAI) & 21 & N.A. & $19 \cdot 19$ & $13 \cdot 87$ \\
\hline Depression (BDI-II) & 21 & N.A. & $23 \cdot 69$ & $12 \cdot 56$ \\
\hline Self-esteem (RSE) & 10 & N.A. & $2 \cdot 37$ & $0 \cdot 70$ \\
\hline Medication adherence (MARS) & 10 & N.A. & $0 \cdot 58$ & $0 \cdot 23$ \\
\hline
\end{tabular}

BAI, Beck Anxiety Inventory; BDI-II, Beck Depression Inventory-II; RSE, Rosenberg Self-Esteem Scale; MARS, Medication Adherence Rating Scale; N.A., not applicable.

a This internal reliability value was derived from the first three items of the Scale of Unawareness of Mental Disorder (SUMD), as items 4 and/or 5 are not applicable to every participant.

standard medium effect size of 0.5 and an $\alpha$ significance level of 0.05 (Cohen, 1992).

\section{RESULTS}

Of the first 137 participants with non-affective psychoses recruited into the PRP trial, 100 $(73 \%)$ completed all measures required to test our hypotheses. The majority of the participants were male $(72 \%)$, single $(68 \%)$, unemployed $(75 \%)$ and white $(68 \%)$. The mean age was $39 \cdot 1$ (S.D. $=10 \cdot 6$ ) years. The mean length of illness (as measured from first contact with mental health services) was 12.4 (s.D. $=9 \cdot 2$ ) years. Symptom severity was rated by independent observers using the positive symptom component of the Positive and Negative Syndrome Scale (PANSS; Kay et al. 1991). The mean PANSS positive symptom score for the sample was $18 \cdot 3$ (s.D. $=5 \cdot 2$ ). The excluded subjects did not differ significantly from the study sample on any of the above measures. The sample consisted of participants presenting with a diagnosis of schizophrenia $(n=84)$, schizoaffective disorder $(n=14)$ and delusional disorder $(n=2)$.

Internal reliability scores (Cronbach, 1951) were calculated for the insight scale, and the three (applicable) illness perceptions constructs of timeline, cure/control and consequences. These were satisfactory (see Table 1).
The mean and standard deviation scores in Table 1 indicate that the sample reported a moderate frequency of psychotic symptoms. Self-reported positive symptoms correlated strongly with observer-rated symptoms. Observer-rated positive symptoms did not correlate significantly with either depression or self-esteem. Although PANSS positive symptoms correlated significantly with anxiety, the relationship was relatively weak $(r=0 \cdot 27)$ compared to subjectively reported symptomatology measured through the 'identity' construct $(r=0 \cdot 59)$. The participants perceived themselves as holding some degree of cure/control, but also appeared to believe that many negative consequences would follow as a direct result of their illness. The respondents varied considerably in their views about the likely duration of their illness. Internal causal attributions were widely endorsed. For example, $68 \%$ agreed that 'My state of mind played a major part in causing my current problems/illness'.

Three attribution items (i.e. a germ or virus/ diet/pollution caused my illness) were endorsed by a very limited proportion of the sample $(5-22 \%)$, and did not appear to be popular aetiological explanations of psychosis. They were therefore excluded from further analysis. As the remaining causal attributions were measured by single items, and were therefore not regarded as continuous variables, these 
Table 2. Correlation analysis (Pearson's $r$ ): positive symptoms, insight, illness perceptions, medication adherence and emotional dysfunction $(n=100)$

\begin{tabular}{|c|c|c|c|c|c|c|c|c|c|}
\hline & PANSS & Insight & MARS & Anxiety & Depression & Self-esteem & Identity & Timeline & Consequences \\
\hline Insight & $-0 \cdot 50 * *$ & & & & & & & & \\
\hline MARS & $-0 \cdot 37 * *$ & $0 \cdot 24 *$ & & & & & & & \\
\hline Anxiety & $0 \cdot 27 * *$ & $-0 \cdot 02$ & $-0 \cdot 25^{*}$ & & & & & & \\
\hline Depression & $0 \cdot 17$ & -0.02 & $-0.33 * *$ & $0 \cdot 58 * *$ & & & & & \\
\hline Self-esteem & $0 \cdot 03$ & $0 \cdot 12$ & $-0 \cdot 26^{* *}$ & $0 \cdot 43 * *$ & $0 \cdot 77 * *$ & & & & \\
\hline Identity & $0 \cdot 46 * *$ & $-0 \cdot 11$ & $-0 \cdot 24^{*}$ & $0 \cdot 59 * *$ & $0 \cdot 56^{* *}$ & $0 \cdot 49^{* *}$ & & & \\
\hline Timeline & $-0 \cdot 01$ & $0 \cdot 10$ & -0.09 & $0 \cdot 27 * *$ & $0 \cdot 47 * *$ & $0 \cdot 40 * *$ & $0 \cdot 25^{*}$ & & \\
\hline Consequences & $0 \cdot 15$ & $0 \cdot 01$ & $-0 \cdot 33^{* *}$ & $0 \cdot 43 * *$ & $0 \cdot 54 * *$ & $0 \cdot 42 * *$ & $0 \cdot 46^{* *}$ & $0 \cdot 42 * *$ & \\
\hline Cure/Control & $-0 \cdot 25^{*}$ & $0 \cdot 29 * *$ & $0 \cdot 29 * *$ & $-0 \cdot 26^{* *}$ & $-0 \cdot 35^{* *}$ & $-0 \cdot 35^{* *}$ & $-0 \cdot 15$ & $-0 \cdot 31^{* *}$ & $-0 \cdot 48^{* *}$ \\
\hline
\end{tabular}

PANSS, Positive and Negative Syndrome Scale; MARS, Medication Adherence Rating Scale.

$* p<0 \cdot 05, * * p<0 \cdot 01$.

were analysed separately from the other IPQ constructs.

The mean level of insight, as rated by the study assessors, was moderate, with considerable variance (see Table 1). Levels of depression, anxiety and self-esteem were moderate (see Table 1), but showed a considerable degree of variation.

Table 2 shows Pearson $r$ correlations between insight and illness perception (predictor variables), medication adherence, and emotional dysfunction (dependent variables). There appears to be little relationship between emotional dysfunction and levels of insight as assessed by the SUMD. However, there are many significant correlations between components of illness perception and aspects of emotional dysfunction. A high symptom score, a longer timeline, a high level of perceived consequences and a low level of cure/control were significantly related to higher levels of depression, and patterns of association with both anxiety and self-esteem were similar. Simultaneous multiple regression analyses were conducted to estimate the proportion of variance accounted for in the dependent variables. Associations between variables that reached the $p<0.05$ level of significance were entered into the three separate regression equations. Illness perception constructs explained a substantial proportion of the variance in depression (46\%), anxiety (36\%) and selfesteem $(34 \%)$. The analyses demonstrated that the proposed illness perception framework produced statistically significant association with anxiety $(F=15 \cdot 0, \mathrm{df}=4, p<0 \cdot 001)$, depression $(F=22.3, \mathrm{df}=4, p<0.001)$ and selfesteem $(F=13 \cdot 5, \mathrm{df}=4, p<0 \cdot 001)$. In anxiety, perceived symptoms emerged as the single significant correlate $(\beta=0.50, p<0 \cdot 001)$. For depression, perceived symptoms $(\beta=0 \cdot 39$, $p<0.001)$, timeline $(\beta=0.25, p<0.01)$ and perceived consequences $(\beta=0.20, p<0.05)$ were all significant. Perceived symptoms $(\beta=0.37$, $p=0.001)$, cure/control $(\beta=-0.19, \quad p<0.05)$ and timeline $(\beta=0.22, p<0.05)$ emerged as the significant variables association with selfesteem.

Table 3 shows Spearman's $\rho$ correlations between the dependent variables and the eight causal attribution items. None of the causal items were significantly related to levels of depression. Attributing one's illness to one's state of mind and to stress was significantly related to higher levels of anxiety and selfesteem respectively. However, caution should be exercised when interpreting these findings because of the possible influence of multiple comparison testing.

\section{The relative contribution of insight, illness perceptions and depression to medication adherence}

Given the hypothesized influence of emotional dysfunction upon adaptive coping in the model of Leventhal et al., depression was tested alongside insight and illness perceptions to determine whether it related to medication adherence. Thus, depression, insight, identity, cure/ control and consequences were entered into the equation. This model explained $16 \%$ of the variance in medication adherence $(F=4 \cdot 8$, $\mathrm{df}=5, p=0.001)$. Although depression and the perceived consequences of illness were the variables most strongly related to self-reported 


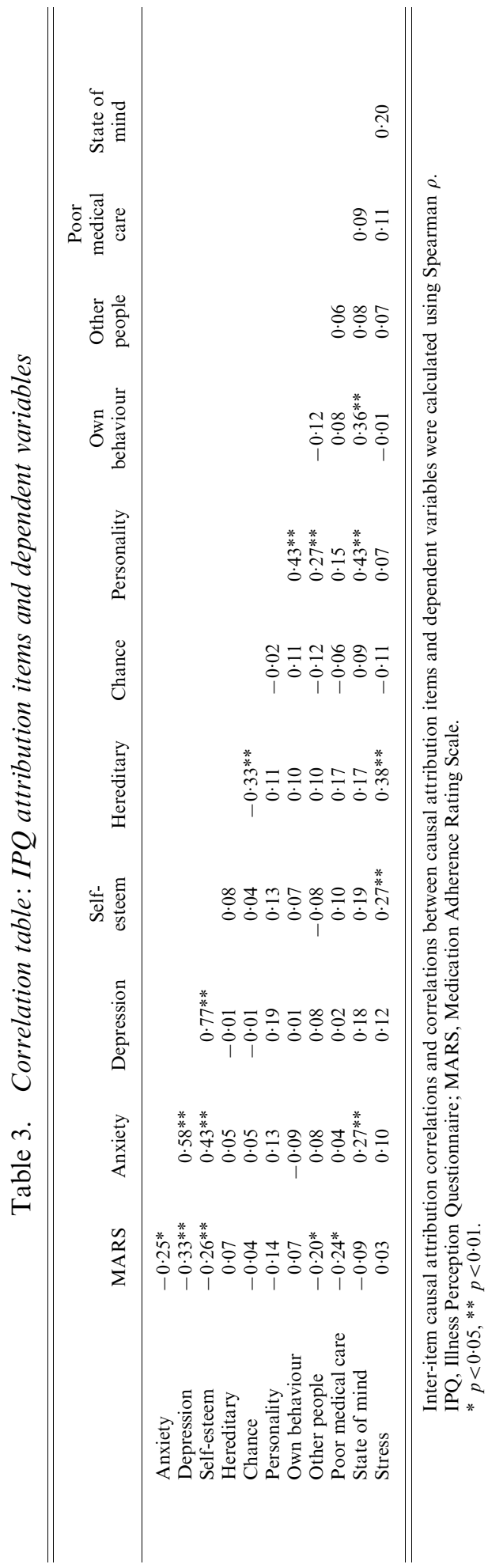

medication adherence in the univariate analysis, only insight $(\beta=0.22, p<0.05)$ emerged as significant in the multiple regression analysis. In a separate analysis, attributions that other people played a role in the cause of illness and attributing illness to poor medical care in the past were significantly and negatively related to self-reported medication adherence.

\section{DISCUSSION}

This study used a cross-sectional design. Such an approach does not, of course, permit interpretation of causality. However, the results of the present study suggest that the level of insight held by the sample was not related to emotional dysfunction. In accordance with our hypotheses, we also found that illness perception constructs of identity, cure/control, consequences and timeline were significantly related to depression, anxiety and self-esteem. Furthermore, specific causal attributions were related to emotional variables. In a series of multivariate analyses, increased levels of perceived consequences of the illness were related to increased depression. A greater level of anxiety was associated with heightened perception of symptoms. Lower self-esteem was related to a weaker perception of cure/control, a longer timeline and a greater perception of symptoms.

The results of our study are in many ways similar to the findings of Lobban et al. (2004) and Jolley \& Garety (2004). Although Lobban et al. did not measure insight and used different measures of depression and anxiety (the Hospital Anxiety and Depression Scale; Zigmond \& Snaith, 1983), they found similar strong relationships between illness perceptions (identity, timeline and consequences) and anxiety and depression. In the present study, a longer timeline perception was positively, and significantly, correlated with depression as in the pilot study of Jolley \& Garety (2004).

Investigation of the relationship between observer-rated positive symptoms, as measured in the PANSS, and perceived symptoms revealed that, while observer-rated and perceived symptoms were correlated positively, it was the perceived (subjective) rather than the observerrated (objective) symptom levels that were more strongly associated with anxiety, depression 
and low self-esteem. This seems to confirm that it is the appraisal of symptoms rather than their occurrence that is distressing. The same may apply to other aspects of illness perception; that is, they too may not be accurate (as perceived by observers) representations of the patient's predicament.

Cognitive behavioural therapy (CBT) for psychosis is a relatively new intervention that aims to reduce the distress associated with positive symptoms of psychosis (Pilling et al. 2002). The findings of this study would, if replicated, provide some pointers for the development of CBT in psychosis. Lengthy timeline perceptions associated with psychosis were related to a greater level of depression. Therapeutic approaches should emphasize that having a psychotic disorder does not necessarily equate to a lifetime of illness, and that steps can be taken to reduce the likelihood of relapse and the chronicity of disability. Indeed, challenging negative beliefs about the controllability of the illness and developing individualized 'recovery action plans' have been shown to promote improved outcome in non-psychiatric conditions (Petrie et al. 2002).

Estimates have suggested that $55 \%$ of patients presenting with chronic psychotic illnesses (e.g. schizophrenia) have significant difficulties adhering to their medication regimen (Fenton et al. 1997). Similar rates of nonadherence have been documented among individuals presenting with a first episode of psychosis (Coldham et al. 2002). Although much research has sought factors predictive of adherence-related behaviour (Pan \& Tantam, 1989; Kampmann \& Lehtinen, 1999; Lacro et al. 2002), and compliance therapy has been effective in improving medication adherence (Kemp et al. 1998), the need to identify specific targets for intervention to enhance our understanding of the cognitive factors that underpin behavioural change has been highlighted (Adams \& Scott, 2000; Scott \& Pope, 2002). Contrary to our second hypothesis, our results suggest that reduced insight was associated significantly with poorer medication adherence. This finding may, in part, be explained by the fact that one facet of the SUMD assessment of insight (Amador et al. 1993) measures perceptions of awareness of the achieved effects of medication. However, the finding that illness perceptions per se did not directly explain a substantial proportion of the variance in adherence is consistent with recent illness perception research. For example, Horne and Weinman (2002) have shown that specific beliefs about treatment (rather than the illness) are the key influences on adherence, and that illness perceptions are related to these treatment beliefs in a logical way, particularly to patients' beliefs about their need for treatment.

Enhancing perceptions of controllability and working with beliefs about the impact of perceived consequences of psychosis are likely to be useful intervention targets, and a longitudinal test could determine whether such a focus also results in improved adherence. As noted earlier, using personalized action plans to tackle perceived barriers to treatment adherence should be considered. Gibson et al. (2000) in a systematic review found that asthma patients in receipt of a personalized action plan were more adherent to medication, were less likely to be hospitalized, and demonstrated improved outcome in comparison to those with no action plan.

Recognizing that our model only accounted for a modest amount of the variance in medication adherence, future research should highlight the importance of beliefs about treatment, particularly beliefs about the negative consequences of medication (Horne \& Weinman, 1999). Moreover, as found by DiMatteo et al. (2000), our results partially suggest that participants with higher levels of depression seem less likely to adhere to their medication regimen.

Our study has a number of limitations. First, because of its cross-sectional design, we cannot infer the direction of causality; a longitudinal study is needed for this, particularly as illness perceptions may improve as symptoms improve. Indeed, the importance of measuring cognitions and outcomes over time is highlighted in recent research by Drake et al. (2004). This prospective study found a positive relationship between insight and depression, but only at onset. Subsequently, they found that paranoia predicted depression as the illness progressed. A second limitation is that the study participants had consented to take part in an intervention trial. The sample we examined may not therefore be representative of the general population of people with psychosis. It may, however, be 
reasonably representative of a group willing to participate in psychological interventions, including adherence therapy. The findings of Lobban et al. (2004) in a different sample were similar, indicating that these results may be generalizable. Finally, although the MARS holds considerable concurrent and construct validity and has satisfied internal and test-retest reliability measures, an additional objective measure of medication adherence would have been desirable.

In conclusion, an awareness of appraisals such as illness perceptions and their interactions with emotional responses may generate improvements in interventions for people with psychosis.

\section{ACKNOWLEDGEMENTS}

We thank the participants and the staff of North East London Mental Health NHS Trust, South London and Maudsley NHS Trust, Norfolk Mental Health NHS Trust and Camden and Islington Mental Health and Social Care NHS Trust for their commitment to the completion of this study. This work was supported by a programme grant from the Wellcome Trust (No. 062452).

\section{DECLARATION OF INTEREST}

None.

\section{REFERENCES}

Adams, J. \& Scott, J. (2000). Predicting medication adherence in severe mental disorders. Acta Psychiatric Scandinavica 101, 119-124.

Addington, D., Addington, J. \& Maticka-Tyndale, E. (1993). Assessing depression in schizophrenia: the Calgary Depression Scale. British Journal of Psychiatry 163, 39-44.

Amador, X. F. \& David, A. S. (1998). Insight and Psychosis. Oxford University Press: New York.

Amador, X. F., Strauss, D. H., Yale, S. A., Flaum, M. M., Endicott, J. \& Gorman, J. M. (1993). Assessment of insight in psychosis. American Journal of Psychiatry 150, 873-879.

Barrowclough, C., Lobban, F., Hatton, C. \& Quinn, J. (2001). Investigation of models of illness in carers of schizophrenic patients using the Illness Perception Questionnaire. British Journal of Clinical Psychology 40, 371-385.

Beck, A. T., Epstein, N., Brown, G. \& Steer, R. A. (1988). An inventory for measuring clinical anxiety: psychometric properties. Journal of Consulting and Clinical Psychology 56, 893-897.

Beck, A. T., Steer, R. A. \& Brown, G. K. (1996). BDI-II Manual. The Psychological Corporation: San Antonio, TX.

Birchwood, M. (2003). Pathways to emotional dysfunction in first-episode psychosis. British Journal of Psychiatry 182, 373-375.
Birchwood, M., Iqbal, Z., Chadwick, P. \& Trower, P. (2000). Cognitive approach to depression and suicidal thinking in psychosis. British Journal of Psychiatry 177, 516-521.

Birchwood, M., Mason, R., MacMillan, F. \& Healy, J. (1993). Depression, demoralization and control over psychotic illness: a comparison of depressed and non-depressed patients with a chronic psychosis. Psychological Medicine 23, 387-395.

Bishop, G. D. \& Converse, S. A. (1986). Illness representations: a prototype approach. Health Psychology 5, 95-114.

Byrne, S., Trower, P., Birchwood, M., Meaden, A. \& Nelson, A. (2003). Command hallucinations: cognitive theory, therapy, and research. Journal of Cognitive Psychotherapy 17, 67-84.

Cohen, J. A. (1992). Power primer. Psychological Bulletin 112, 155-159.

Coldham, E. L., Addington, J. \& Addington, D. (2002). Medication adherence of individuals with a first episode of psychosis. Acta Psychiatrica Scandinavica 106, 286-290.

Cooke, M., Kuipers, E. \& Kumari, V. (2005). Disease, deficit or denial? Models of poor insight in psychosis. Acta Psychiatrica Scandinavica 112, 4-17.

Cronbach, L. J. (1951). Coefficient alpha and the internal structure of tests. Psychometrika 16, 297-334.

David, A. S. (1998). The clinical importance of insight. In Insight and Psychosis (ed. X. F. Amador and A. S. David), pp. 332-351. Oxford University Press: New York.

DiMatteo, M. R., Lepper, H. S. \& Croghan, T. W. (2000). Depression is a risk factor for noncompliance with medical treatment. Metaanalysis of the effects of anxiety and depression on patient adherence. Archives of Internal Medicine 160, 2101-2107.

Drake, R. J., Pickles, A., Bentall, R. P., Kinderman, P., Haddock, G., Tarrier, N. \& Lewis, S. W. (2004). The evolution of insight, paranoia, and depression during early schizophrenia. Psychological Medicine 34, 285-292.

Fenton, W. S., Blyer, C. R. \& Heinssen, R. K. (1997). Determinants of medication compliance in schizophrenia: empirical and clinical findings. Schizophrenia Bulletin 23, 637-651.

Freeman, D. \& Garety, P. A. (2003). Connecting neurosis and psychosis: the direct influence of emotion on delusions and hallucinations. Behaviour Research and Therapy 41, 923-947.

Freeman, D., Garety, P. A., Bebbington, P. E., Smith, B., Rollinson, R., Fowler, D., Kuipers, E., Ray, K. \& Dunn, G. (2005). Psychological investigation of the structure of paranoia in a non-clinical population. British Journal of Psychiatry 186, 427435.

Garety, P. A., Kuipers, E., Fowler, D., Freeman, D. \& Bebbington, P. E. (2001). A cognitive model of the positive symptoms of psychosis. Psychological Medicine 31, 189-195.

Ghaemi, S. N. \& Pope, H. G. (1994). Lack of insight in psychotic and affective disorders: a review of empirical studies. Harvard Review of Psychiatry 2, 22-33.

Gibson, P. G., Coughlan, J., Wilson, A. J., Abramson, M., Bauman, A., Hensley, M. J. \& Walters, E. H. (2000). Self-management education and regular practitioner review for adults with asthma. Cochrane Database Systematic Review 2. Art. no. CD001117.

Hagger, M. \& Orbell, S. (2003). A meta-analytic review of the common-sense model of illness representations. Psychology and Health 18, 141-184.

Hogan, T. P., Awad, A. G. \& Eastwood, R. (1983). A self-report scale predictive of drug compliance in schizophrenics: reliability and discriminative validity. Psychological Medicine 13, 177-183.

Horne, R. \& Weinman, J. (1999). Patients' beliefs about prescribed medicines and their role in adherence to treatment in chronic physical illness. Journal of Psychosomatic Research 47, 555-567.

Horne, R. \& Weinman, J. (2002). Self-regulation and selfmanagement in asthma: exploring the role of illness perceptions and treatment beliefs in explaining non-adherence to preventer medication. Psychology and Health 17, 17-32.

Iqbal, Z., Birchwood, M., Chadwick, P. \& Trower, P. (2000). Cognitive approach to depression and suicidal thinking in psychosis: 2. Testing the validity of a social ranking model. British Journal of Psychiatry 177, 522-528. 
Johns, S. L. \& van Os, J. (2001). The continuity of psychotic experience in the general population. Clinical Psychology Review 21, 1125-1141.

Jolley, S. \& Garety, P. A. (2004). Insight and delusions, a cognitive psychological approach. In Insight and Psychosis (ed. X. F. Amador and A. S. David), pp. 89-100. Oxford University Press: New York.

Kampmann, O. \& Lehtinen, K. (1999). Compliance in psychoses. Acta Psychiatrica Scandinavica 100, 167-175.

Kay, S. R., Opler, L. A. \& Fiszbein, A. (1991). PANSS Positive and Negative Syndrome Scale Rating Manual. Multi-Health Systems Inc.: Toronto.

Kemp, R., Kirov, G., Everitt, B., Hayward, P. \& David, A. (1998). A randomised controlled trial of compliance therapy: 18-month follow-up. British Journal of Psychiatry 172, 413-419.

Lacro, J. P., Dunn, L. B., Dolder, C. R., Leckband, S. G. \& Jeste, D. V. (2002). Prevalence of and risk factors for medication nonadherence in patients with schizophrenia: a comprehensive review of recent literature. Journal of Clinical Psychiatry 63, 892-909.

Leventhal, H., Nerenz, D. R. \& Steele, D. F. (1984). Illness representations and coping with health threats. In Handbook of Psychology and Health, vol. IV (ed. A. Baum and J. Singer), pp. 219-252. Erlbaum: Hillsdale, NJ.

Lobban, F., Barrowclough, C. \& Jones, S. (2003). A review of the role of illness models in severe mental illness. Clinical Psychology Review 23, 171-196.

Lobban, F., Barrowclough, C. \& Jones, S. (2004). The impact of beliefs about mental health problems and coping on outcome in schizophrenia. Psychological Medicine 37, 1165-1174.

Morisky, D. E., Green, L. W. \& Levine, D. M. (1986). Concurrent and predictive validity of a self-reported measure of medication adherence. Medical Care 24, 67-74.
Morrison, A. P. (2001). The interpretation of intrusions in psychosis: an integrative cognitive approach to hallucinations and delusions. Behavioural and Cognitive Psychotherapy 29, 257-276.

Pan, P. C. \& Tantam, D. (1989). Clinical characteristics, health beliefs and compliance with maintenance treatment: a comparison between regular and irregular attenders at a depot clinic. Acta Psychiatrica Scandinavica 79, 564-570.

Petrie, K. J., Cameron, L., Ellis, C. J., Buick, D. \& Weinman, J. (2002). Changing illness perceptions after myocardial infarction: an early intervention randomised controlled trial. Psychosomatic Medicine 64, 580-586.

Pilling, S., Bebbington, P., Kuipers, E., Garety, P., Geddes, J., Orbach, G. \& Morgan, C. (2002). Psychological treatments in schizophrenia: I. Meta-analysis of family intervention and cognitive behaviour therapy. Psychological Medicine 32, 763-782.

Rathod, S., Kingdon, D. G., Turkington, D. \& Smith, P. (2005) Insight into schizophrenia: the effects of cognitive behavioural therapy on the components of insight and association with sociodemographics-data on a previously published randomised controlled trial. Schizophrenia Research, 74, 211-219.

Rosenberg, M. (1965). Society and the Adolescent Self-Image. Princeton University Press: Princeton, NJ.

Scott, J. \& Pope, M. (2002). Non-adherence with mood stabilizers: prevalence and predictors. Journal of Clinical Psychiatry 63, 384-391.

Thompson, K., Kulkarni, J. \& Sergejew, A. A. (2000). Reliability and validity of a new Medication Adherence Rating Scale (MARS) for the psychoses. Schizophrenia Research 42, 241-247.

Weinman, J., Petrie, K., Moss-Morris, R. \& Horne, R. (1996). The Illness Perception Questionnaire: a new method for assessing the cognitive representation of illness. Psychology and Health 11, 431-445.

Zigmond, A. S. \& Snaith, R. P. (1983). The Hospital and Anxiety and Depression Scale. Acta Psychiatrica Scandinavica 67, 361-370. 